\title{
Dichotomy of the $\boldsymbol{H}$-Quasi-cover problem *
}

\author{
Jiří Fiala ${ }^{\star \star}$ and Marek Tesař ${ }^{\star \star \star}$ \\ Department of Applied Mathematics and DIMATIA, Charles University, \\ Malostranské nám. 25, 11800 Prague, Czech Republic \\ \{fiala, tesar\}@kam.mff.cuni.cz
}

\begin{abstract}
We show that the problem whether a given simple graph $G$ admits a quasi-covering to a fixed connected graph $H$ is solvable in polynomial time if $H$ has at most two vertices and that it is NP-complete otherwise.

As a byproduct we show constructions of regular quasi-covers and of multi-quasi-covers that might be of independent interest.
\end{abstract}

Keywords: Computational complexity, dichotomy, graph cover.

\section{Introduction}

A homomorphism between two graphs $G$ and $H$ is an edge preserving mapping $f: V(G) \rightarrow V(H)$. We focus on homomorphisms $f$ that satisfy local constraints. For instance it might be required for each vertex $u$ of $G$ that all neighbors of its image $f(u)$, are used when the mapping $f$ is restricted on the neighborhood of $u$, formally $\left|f^{-1}(v) \cap N_{G}(u)\right| \geq 1$ for each $v \in N_{H}(f(u))$. In other words $f$ should act surjectively between $N_{G}(u)$ and $N_{H}(f(u))$ for each $u \in V(G)$. In such a situation we say that $f$ is a locally surjective homomorphism.

We focus in a particular case of locally surjective homomorphisms, called quasi-coverings. These satisfy that for every vertex $u$ of $G$ there exists a positive integer $c$ such that $\left|f^{-1}(v) \cap N_{G}(u)\right|=c$ for every $v \in N_{H}(f(u))$ - in such a case we say that $\left.f\right|_{N_{G}(u)}$ is $c$-fold between $N_{G}(u)$ and $N_{H}(f(u))$. Note that the constant $c$ may vary for different vertices of $G$. If such a quasi-covering projection from $G$ to $H$ exists, we say that $G$ quasi-covers $H$ or that $G$ is a quasi-cover of $H$.

Locally surjective homomorhisms and quasi-covers are closely related to homomorphisms that are locally injective (bijective, resp.), i.e. those edgepreserving mappings satisfying that for every vertex $u$ it holds that $N_{G}(u)$ is mapped to $N_{H}(f(u))$ injectively (bijectively, resp.). Locally bijective homomorphisms are also known as covering projections. Similarly, locally injective homomorphisms are sometimes called partial covering projections, while locally surjective homomorphisms are also known as role assignments.

* Supported by Charles University as GAUK 95710

** Supported by MŠMT ČR grant LH12095 and GAČR grant P202/12/G061

$\star \star \star$ Supported by the grant SVV-2012-265313 
Covering — primal embedding Quasi-covering — dual embedding
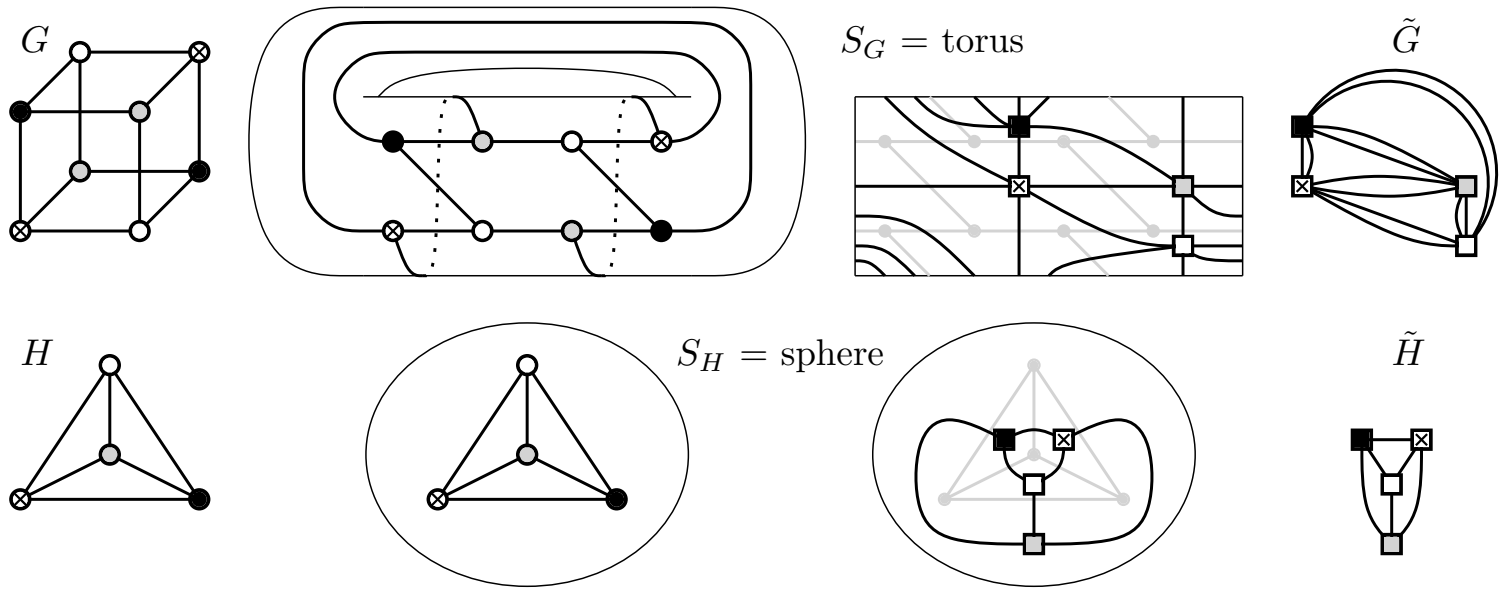

$\tilde{H}$

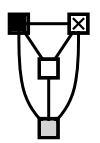

Fig. 1. Example of obtaining a quasi-covering from $G=Q_{3}$ that covers $H=K_{4}$. The mappings are indicated by vertex colors.

Covers and quasi-covers are discrete variants of the corresponding notions in algebraic topology. To obtain a quasi-cover consider a 2-cell embedding of a graph $H$ in an orientable surface $S_{H}$ and a graph $G$ covering $H$ via $f$ (see Figure 1 for an example). By using an 2-cell embedding of $G$ where every vertex $u$ uses the same neighbor ordering as $f(u)$, we obtain a surface $S_{G}$ with the following property: the covering $f$ extends to a mapping between $S_{G}$ and $S_{H}$ which respects edges and faces of both embeddings. In addition this mapping is a local homeomorphism except of those faces whose length is a multiple of the length of its image (the length is measured in the number of vertices on the face). The mapping on these faces contains singularity of degree being equal to the ratio of the two face lengths.

We construct duals $\tilde{G}$ and $\tilde{H}$ from the two 2-cell embeddings of $G$ and $H$ in $S_{G}$ and $S_{H}$, resp. and factor the mapping between $S_{G}$ and $S_{H}$ to a homomorphism between $\tilde{G}$ and $\tilde{H}$. Moreover, as the boundary of each face of $G$ (in $S_{G}$ ) must be mapped homeomorphically onto the boundary of the appropriate face of $H$ (in $S_{H}$ ), we get that the resulting mapping between duals $\tilde{G}$ and $\tilde{H}$ is $c$-fold on the neighborhood of any vertex of $\tilde{G}$.

We follow the usual scenario for the question whether a graph $G$ admits a possibly specific homomorphism to $H$. Since such tests allow no simple criterion, we define several classes of decision problems: $H$-Hom, $H$-QCover, $H$-LIHOM, $H$-LSHom, and $H$-LBHom, resp. In all of them $H$ is a fixed target graph and the query is whether a graph $G$ on the input admits a homomorphism to $H$ of the appropriate constraint: being a homomorphism, a quasi-covering, locally injective, locally surjective, and locally bijective, resp.

The computational complexity of $H$-Hom was fully determined by Hell and Nešetřil [11]. They show that the problem is solvable in polynomial time only for bipartite $H$ and that it is NP-complete otherwise. 
The study of $H$-LSHom was initiated by Kristiansen and Telle [16] and a full dichotomy was completed by Fiala and Paulusma [9]. For connected $H$ they showed that $H$-LSHOM is NP-complete whenever $H$ has at least three vertices; for disconnected $H$ the condition is more elaborate.

The complexity of locally bijective homomorphisms was first studied by Bodlaender [3] and by Abello et al. [1]. Despite the subsequent effort of several authors (see e.g. papers by Kratochvíl et al. $[13,14,15]$ or a survey by Fiala and Kratochvíl [8]) the complete characterization has not been settled yet.

The dichotomy for the computational complexity of the $H$-LIHom problem is also not known. Some partial results can be found in $[4,5,6,17,2]$. It might be of independent interest that locally injective homomorphisms generalize the notion of $L(2,1)$-labelings, which are motivated by the frequency assignment problem. Fiala and Kratochvíl [7] also considered the list version of the $H$-LIHom problem and provided here a dichotomy.

In our paper we show that the $H$-QCOVER problem yields for connected graphs $H$ the same dichotomy as the $H$-LSHom problem:

Theorem 1. Let $H$ be a connected graph. If $H$ has at least three vertices, then the $H$-QCover problem is NP-complete. Otherwise, it is solvable in linear time.

This is in contrast with the well known fact that testing the existence of a covering between two embedded graphs that locally extends to a homeomorphism of the embedding admits a straightforward quadratic-time algorithm: if the mapping is determined for any edge, it has a unique extension to adjacent edges given by the ordering of the edges around a vertex in the embedding. Therefore also the corresponding problem for the quasi-coverings between the associated duals is polynomially solvable with the same time complexity.

\section{Preliminaries}

In this paper we consider only simple and connected graphs. We denote the set of vertices of a graph $G$ by $V(G)$ and its edge set by $E(G)$. We denote the degree of a vertex $v$ in $G$ by $\operatorname{deg}_{G}(v)$ and the set of all neighbors of $v$ - the neighborhood of $v$ - by $N_{G}(v)$. In a $d$-regular graph all vertices are of the same degree $d$.

For the definition of other standard graph theoretic terms (like paths, complete bipartite graphs), see e.g. a monograph by Nešetřil and Matoušek [18].

We call a mapping $f: X \rightarrow Y$ between two sets $c$-fold if for all $y \in Y$ it holds that $\left|f^{-1}(y)\right|=c$.

Recall that a homomorphism $f: G \rightarrow H$ is a quasi-covering if for each vertex $v \in V(G)$ there exists an integer $c$ such that $\left.f\right|_{N_{G}(v)}$ is $c$-fold between $N_{G}(v)$ and $N_{H}(f(v))$. Note that quasi-covering which is 1 -fold on every vertex of $G$ is indeed a covering projection.

Observe that the composition of a $c$-fold and a $d$-fold mapping is a $c d$-fold mapping. Hence a composition of two quasi-coverings is also a quasi-covering. 
We use this fact also in the case when one of these two mappings is a covering projection or an automorphism.

By a boundary $\delta H$ of an induced subgraph $H$ of a graph $G$ we mean the set of vertices of $H$ that are adjacent to a vertex outside $H$.

The symbol lcmd $(G)$ stands for the least common multiple of degrees of all non-isolated vertices in $G$.

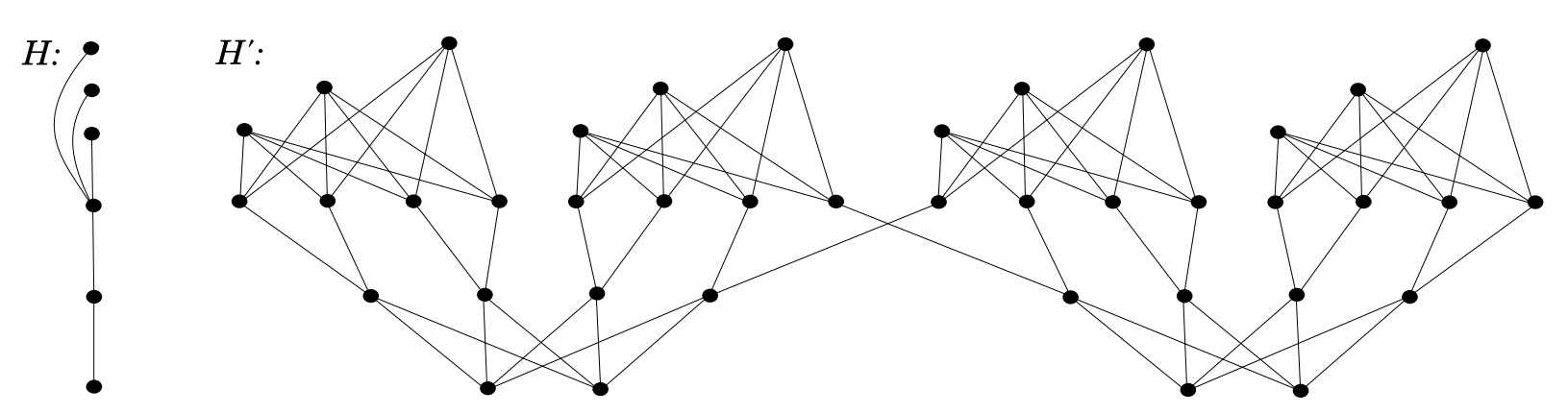

Fig. 2. Construction of the graph $H^{\prime}$ for a graph $H$ with $d=4$. Copies of vertices of graph $H$ are in horizontal lines

Proposition 1. For every graph $H$ there exists a regular connected graph $H^{\prime}$ such that $H^{\prime}$ quasi-covers $H$.

Proof. Without loss of generality assume that $E(H)$ is not empty, as otherwise $H$ itself is 0-regular and $H^{\prime}$ could be chosen to consist of a single isolated vertex. Let $d=\operatorname{lcmd}(H)$. We construct a $d$-regular graph $H^{\prime}$ and quasi-covering $h: H^{\prime} \rightarrow H$ as follows.

For every vertex $x \in V(H)$ we insert into $V\left(H^{\prime}\right)$ vertices $x_{1}, x_{2}, \ldots, x_{d \operatorname{deg}_{H}(x)}$. All these $d \operatorname{deg}_{H}(x)$ vertices are mapped onto $x$ by $h$ (see Figure 2). For every edge $x y \in E(H)$ we add $d^{2}$ edges between sets $h^{-1}(x)$ and $h^{-1}(y)$ in such a way that every $x_{i} \in h^{-1}(x)$ is incident with $\frac{d}{\operatorname{deg}_{H}(x)}$ of these $d^{2}$ edges. Analogously every $y_{i} \in h^{-1}(y)$ is incident with $\frac{d}{\operatorname{deg}_{H}(y)}$ of them. This can be done e.g. by using $\operatorname{deg}_{H}(x) \operatorname{deg}_{H}(y)$ copies of the complete bipartite graph $K_{\operatorname{deg}_{H}(y)}, \frac{d}{\operatorname{deg}_{H}(x)}$.

If $H^{\prime}$ is not connected, we restrict $H^{\prime}$ to any of its connected component containing at least one edge. The obtained graph $H^{\prime}$ is $d$-regular since for every $x_{i} \in V\left(H^{\prime}\right)$ it holds that $\operatorname{deg}_{H^{\prime}}\left(x_{i}\right)=\operatorname{deg}_{H}(x) \frac{d}{\operatorname{deg}_{H}(x)}=d$.

By the construction, for every neighbor $v$ of $h\left(x_{i}\right)=x$ in $H$ we have that $\left|h^{-1}(v) \cap N_{H^{\prime}}\left(x_{i}\right)\right|=\frac{d}{\operatorname{deg}_{H}(x)}$. Therefore, $h$ is $\frac{d}{\operatorname{deg}_{H}(x)}$-fold between $N_{H^{\prime}}\left(x_{i}\right)$ and $N_{H}(x)$, i.e. a quasi-covering as required.

Kratochvíl, Proskurowski, and Telle [13] proved existence of a cover with a special property, which we also use in our paper.

Proposition 2 ([13]). For every d-regular conected graph $H^{\prime}$, there exists a d-regular graph $A$ with a specified vertex a, such that any bijective mapping between $N_{A}(a)$ and $N_{H^{\prime}}\left(x_{i}\right)$ for arbitrary $x_{i} \in V\left(H^{\prime}\right)$ can be extended to a covering projection $g: A \rightarrow H^{\prime}$ satisfying $g(a)=x_{i}$. 
We use the Proposition 2 to construct an analogous graph, called multi-quasicover of $H$ as follows:

Lemma 1. Let $H$ be a connected graph and let $d=\operatorname{lcmd}(H)$. There exists a d-regular graph $A$ with specified vertex a, such that for any non-isolated vertex $x \in V(H)$ it holds that any $\frac{d}{\operatorname{deg}_{H}(x)}$-fold mapping $\varphi: N_{A}(a) \rightarrow N_{H}(x)$ can be extended to a quasi-covering $f: A \rightarrow H$, such that $f(a)=x$.

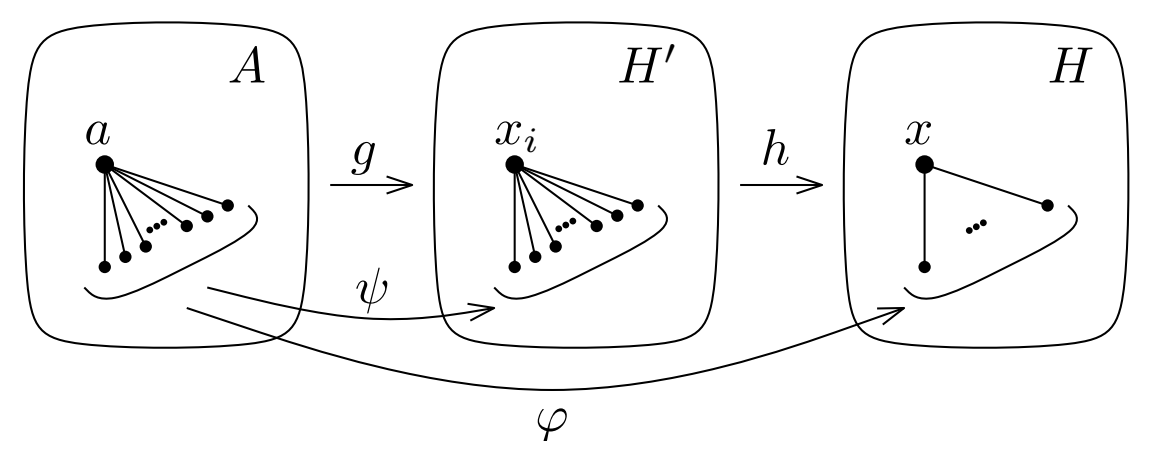

Fig. 3. Construction of multi-quasi-cover $A$ of $H$.

Proof. According to Proposition 1 we first construct a $d$-regular graph $H^{\prime}$ and a quasi-covering $h: H^{\prime} \rightarrow H$. Then we use Proposition 2 for $H^{\prime}$ and obtain the desired $d$-regular graph $A$ with a specified vertex $a$ (see Figure 3).

For the given $x \in V(H)$ and $\frac{d}{\operatorname{deg}_{H}(x)}$-fold mapping $\varphi: N_{A}(a) \rightarrow N_{H}(x)$ we choose arbitrarily $x_{i} \in h^{-1}(x)$ and determine a bijective mapping $\psi: N_{A}(a) \rightarrow$ $N_{H^{\prime}}\left(x_{i}\right)$ such that $h \circ \psi=\varphi$. Such $\psi$ exists since both $\varphi$ and $\left.h\right|_{N_{H^{\prime}}\left(x_{i}\right)}$ are $\frac{d}{\operatorname{deg}_{H}(x)}{ }^{-}$ fold, hence it suffices to match arbitrarily vertices of $\varphi^{-1}(y)$ and $h^{-1}(y) \cap N_{H^{\prime}}\left(x_{i}\right)$ for each neighbor $y$ of $x$.

Let $g$ be the extension of $\psi$ according to Proposition 2. Then $f=h \circ g$ is a composition of two quasi-coverings, i.e. a quasi-covering as well. Since $\left.g\right|_{N_{A}(a)}=$ $\psi$, we get that $\left.f\right|_{N_{A}(a)}=\left.h \circ g\right|_{N_{A}(a)}=h \circ \psi=\varphi$, i.e. $f$ extends $\varphi$. Finally, $f(a)=h(g(a))=h\left(x_{i}\right)=x$ as required.

We involve arguments already used by Fiala and Paulusma [9].

Let $N_{G}^{d}(u)$ be the set of vertices at distance at most $d$ from $u$ in the graph $G$. By induction on $d$ one gets:

Observation 1 If $f: G \rightarrow H$ is locally surjective homomorphism then $f$ is also a surjective mapping between sets $N_{G}^{d}(u)$ and $N_{H}^{d}(f(u))$ for any $u \in V(G)$ and any $d$.

Definition 1. We say that $x$ is a maximal distance vertex in a connected graph $H$, if there exists a vertex $z \in V(H)$ such that the distance between $x$ and $z$ attains the maximum among distances between all possible pairs of vertices in $H$. This maximum distance is called the diameter of $H$, and is denoted by $\operatorname{diam}(H)$. 
Observation 1 provides the following corollaries:

Corollary 1 ([9]). Let $H$ be a graph and let $x$ be a maximal distance vertex in $H$. If $G$ contains $H$ as an induced subgraph such that $\delta H=\{x\}$, then any locally surjective homomorphism $f: G \rightarrow H$ has the property that $f$ restricted to $H$ is an automorphism of $H$.

Corollary 2. Let $H$ be a graph, $x$ be its maximal distance vertex, and let $M$ be the set of vertices at distance $\operatorname{diam}(H)$ from $x$. If $G$ contains $H$ as an induced subgraph such that $\delta H \subseteq M$ then any locally surjective homomorphism $f: G \rightarrow$ $H$ satisfying that $f(x)$ is a maximal distance vertex, has the property that $f$ restricted to $H$ is an automorphism of $H$.

Proof. By the choice of $x$ we get that $\left|N_{G}^{\operatorname{diam}(H)}(x)\right|=\left|N_{H}^{\operatorname{diam}(H)}(f(x))\right|=\left|V_{H}\right|$. A surjective mapping between sets of the same size is a bijection.

\section{Coloring gadgets}

For the purpose of our NP-hardness reductions we build a specific gadget according to the following needs:

Definition 2. Let $H$ be a connected graph and let $x$ be its vertex of degree $k \geq 1$. We say that the graph $F=\mathrm{CG}_{H}(x, m)$ with $m$ specified vertices $u_{1}, \ldots, u_{m}$ is a coloring gadget for $H$ of size $m$ and for $k$ colors if it has the following properties:

- F allows at least one quasi-covering $f: F \rightarrow H$ that maps all specified vertices $u_{i}$ to $x$,

- whenever a graph $G$ contains $F$ as an induced subgraph with $\delta F \subseteq\left\{u_{1}, \ldots\right.$, $\left.u_{m}\right\}$ and whenever $f: G \rightarrow H$ is a quasi-covering, then

i) $f$ restricted to $F$ is a quasi-covering projection as well,

ii) $\operatorname{deg}_{H}\left(f\left(u_{1}\right)\right)=k$,

iii) $N_{H}\left(f\left(u_{i}\right)\right)=N_{H}\left(f\left(u_{1}\right)\right)$ for each specified vertex $u_{i}$

In this section we show that a coloring gadget exists for every connected graph $H$ on at least three vertices.

Lemma 2. Let $H$ be a connected graph on at least three vertices whose all maximal distance vertices are of degree one. Then, for any neighbor $x$ of a maximal distance vertex and any positive integer $m$ the $\mathrm{CG}_{H}(x, m)$ exists.

In particular, the above lemma applies on every path or a tree on at least three vertices.

Proof. Let $z_{1}$ be a maximal distance vertex in $H$, let $x$ be its neighbor, and let $y$ be a vertex at the maximal distance from $z_{1}$. Let $z_{2}, \ldots, z_{t}$ be the neighbors of $x$ other than $z_{1}$ that are also at the maximal distance from $y$ (see Figure 4 ).

We take $m+2$ copies $H_{1}, \ldots, H_{m+2}$ of the graph $H$ and merge all copies of $y$ into a new vertex $w$. Then, we merge the first $m+1$ copies of each $z_{i}$ into a new 

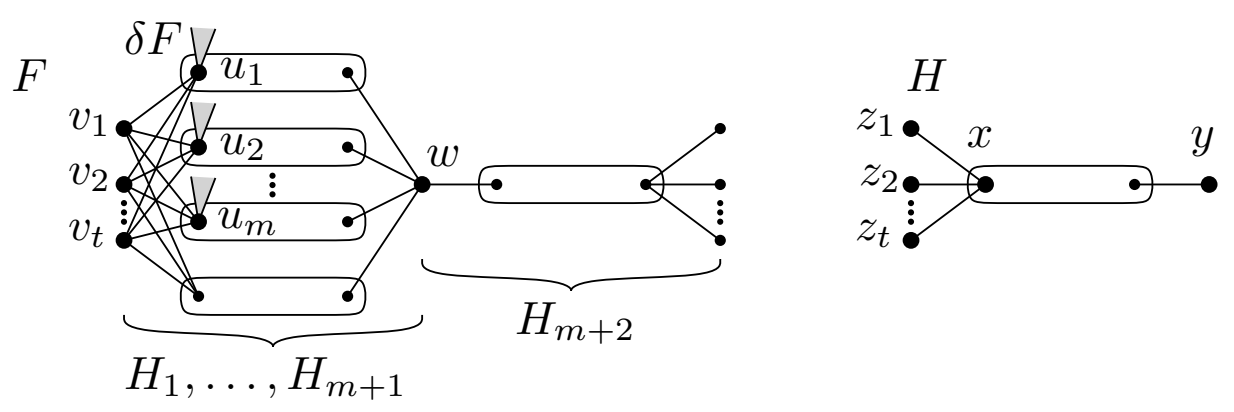

Fig. 4. The coloring gadget $F$ for $H$ with all maximal distance vertices of degree one.

vertex $v_{i}$, and obtain the coloring gadget $F$. For specified vertices $u_{1}, \ldots, u_{m}$ we choose the first $m$ copies of $x$.

A quasi-covering $F \rightarrow H$ can be obtained if we project each $H_{i}$ onto $H$. It means that to show that $F$ is a coloring gadget we only need to prove the conditions $i$ ), $i i$ ), and $i i i$ ) from the Definition 2. Assume that $F$ is an induced subgraph of $G$, such that $\delta F \subseteq\left\{u_{1}, \ldots, u_{m}\right\}$ and that $f: G \rightarrow H$ is a quasicovering.

Since $H_{m+2}$ is an induced subgraph of $G$ with boundary $\delta H_{m+2}=w$, we apply Corollary 1 and get that $f(w)$ is a maximal distance vertex in $H$ and also that $\left.f\right|_{H_{m+2}}$ is an isomorphism to $H$.

We split $w$ back into $m+2$ vertices $w_{1}, \ldots, w_{m+2}$. Denote the resulting graph by $G^{\prime}$. We also alter $f$ on the new vertices $w_{1}, \ldots, w_{m+2}$, which we map onto $f(w)$, The resulting mapping is denoted by $f^{\prime}$. Since $f(w)$ is a maximal distance vertex in $H$, it has a unique neighbor. Hence $f^{\prime}$ is a 1-fold on each $N_{G^{\prime}}\left(w_{i}\right)$, i.e. a quasi-covering $G^{\prime} \rightarrow H$.

We focus on the copy $H_{m+1}$ in $G^{\prime}$ and apply Corollary 2 with respect to $f^{\prime}$ and obtain that $v_{1}, \ldots, v_{t}$ are mapped on maximum distance vertices of $H$. Since maximum distance vertices have unique neighbor and $f$ coincides with $f^{\prime}$ on $N_{G}\left(v_{1}\right)$, we get that $f\left(u_{1}\right)=\cdots=f\left(u_{m}\right)$ and moreover $\operatorname{deg}_{H}\left(f\left(u_{1}\right)\right)=\operatorname{deg}_{H}(x)$. This shows that conditions $i$ ) and iii) from the definition of coloring gadget hold.

By the construction of $F$ and by the fact that $f(w)$ and $y$ can be exchanged by an automorphism of $H$ we get that for each $i \in\{1, \ldots, m\}$ it holds that $\left|N_{G^{\prime}}^{\operatorname{diam}(H)-1}\left(w_{i}\right)\right|=\left|N_{H}^{\operatorname{diam}(H)-1}(f(w))\right|$. By Observation 1 we get that both $\left.f^{\prime}\right|_{H_{i}}$ and $\left.f\right|_{H_{i}}$ are bijections between $V\left(H_{i}\right)$ and $V(H)$. This means that neighbors of $u_{i}$ inside the copy $H_{i}$ must be mapped to $\operatorname{deg}_{H}(x)$ distinct neighbors of $f\left(u_{1}\right)$ in $H$. Hence $\left.f\right|_{H_{i}}$ is an isomorphism between $H_{i}$ and $H$. Therefore, $\left.f\right|_{F}$ is a quasi-covering and the condition $i$ ) holds.

Lemma 3. Let $H$ be a connected graph with a maximal distance vertex $x$ of degree at least two. For every positive integer $m$ a coloring gadget $\mathrm{CG}_{H}(x, m)$ exists.

Proof. Let $k=\operatorname{deg}_{H}(x)$ and $d=\frac{\operatorname{lcmd}(H)}{k}$. 
To construct $F=\mathrm{CG}_{H}(x, m)$ we first take $(m+1) d$ mutually disjoint copies of $H$ and denote them $H_{i}^{t}$ with $i \in\{1, \ldots, m+1\}$ and $t \in\{1, \ldots, d\}$. Intuitively, the symbol $x_{i}^{t}$ will denote the vertex of $H_{i}^{t}$ corresponding to $x$ in $H$.

Separately we construct a $d k$-regular multi-quasi-cover $A$ of $H$ with a specified vertex $a$ according to Lemma 1 . Denote the $d k$ neighbors of $a$ in $A$ by $w_{j}^{t}$ where $j \in\{1, \ldots, k\}$ and $t \in\{1, \ldots, d\}$. We now remove the vertex $a$ from $A$ to obtain the graph $B$.

In the next step we insert into $F$ the disjoint union of $m+1$ copies $B_{1}, \ldots$, $B_{m+1}$ of the graph $B$ (see Figure 5). For every $j \in\{1, \ldots, k\}$ and $t \in\{1, \ldots, d\}$ we merge all $m+1$ copies of the vertex $w_{j}^{t}$ in $B_{1}, \ldots, B_{m+1}$ into a single vertex $v_{j}^{t}$.

We finalize the construction of the graph $F$ by adding edges $x_{i}^{t} v_{j}^{t}$ for all $i \in\{1, \ldots, m+1\}, j \in\{1, \ldots, k\}$, and $t \in\{1, \ldots, d\}$.

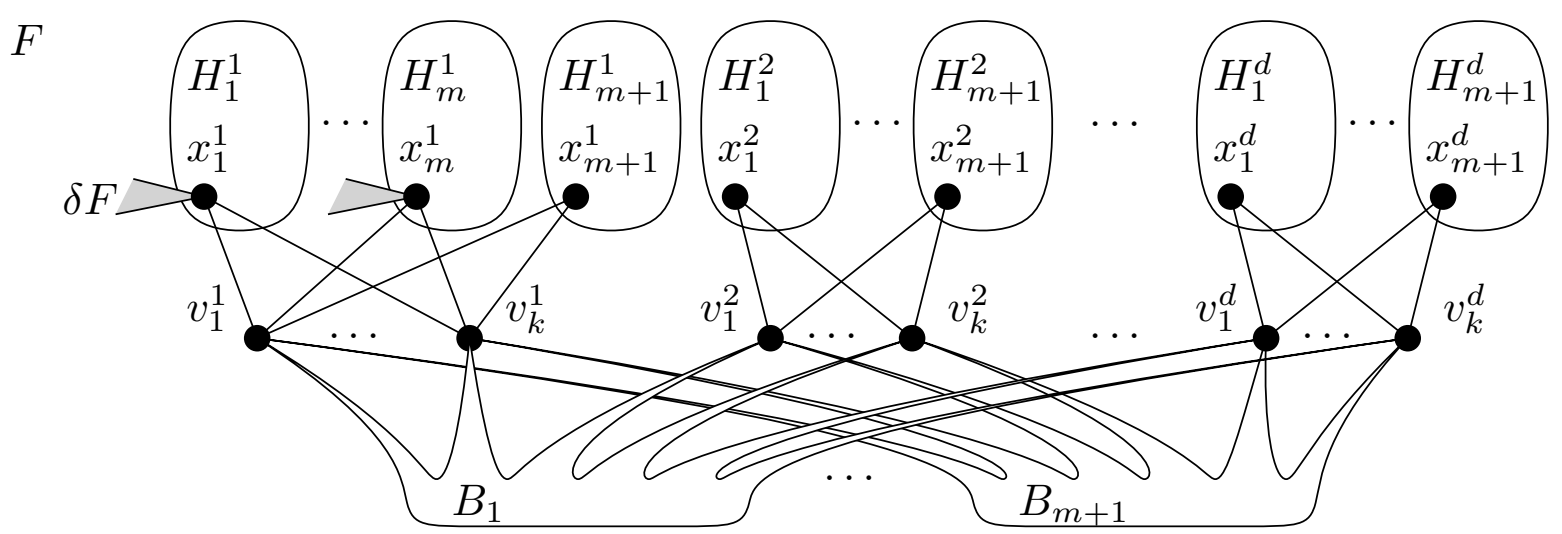

Fig. 5. Example of the construction of $C G_{H}(x, m)$ for maximal vertex $x$ of degree $k \geq 2$.

For the $m$ specified vertices $u_{1}, \ldots, u_{m}$ of the coloring gadget we use the vertices $x_{1}^{1}, \ldots, x_{m}^{1}$.

To show that $F$ quasi-covers $H$ we define a quasi-covering $f: F \rightarrow H$ as follows:

- on every $H_{i}^{t}$ let $f$ act as an isomorphism to $H$, such that $f\left(x_{i}^{t}\right)=x$,

- let $f$ act as a bijection between $v_{1}^{t}, v_{2}^{t}, \ldots, v_{k}^{t}$ and $N_{H}(x)$ for each $t$,

- since the so far defined mapping $f$ is $d$-fold between each $\delta B_{i}$ and $N_{H}(x)$ (and all the neighbors of vertices in $\delta B_{i}$ out of $B_{i}$ are mapped to $x$ ) we may extend it to a quasi-covering inside each subgraph $B_{i}$ according to Lemma 1.

Note that the quasi-covering $f_{A}: A \rightarrow H$ obtained by Lemma 1 is $\frac{d k}{\operatorname{deg}_{H}(y)}$ fold between $N_{A}\left(w_{j}^{t}\right)$ and $N_{H}(y)$, where $y=f_{A}\left(w_{j}^{t}\right)$. Hence, the mapping $f$ is $\frac{(m+1) d k}{\operatorname{deg}_{H}(y)}$-fold between $N_{A}\left(v_{j}^{t}\right)$ and $N_{H}(y)$, i.e. a quasi-covering.

Assume now that $F$ is an induced subgraph of $G$ that allows a quasi-covering $f: G \rightarrow H$ and such that $\delta F \subseteq\left\{x_{1}^{1}, \ldots, x_{m}^{1}\right\}$. We show that conditions $\left.\left.i\right), i i\right)$ and iii) from the Definition 2 hold. Since $x$ is a maximal distance vertex, Corollary 1 
yields that $f$ restricted to each $H_{i}^{1}$ is an isomorphism of $H_{i}^{1}$ and $H$. Hence $\operatorname{deg}_{H}\left(f\left(x_{i}^{1}\right)\right)=\operatorname{deg}_{H}(x)=k$ for each $i \in\{1, \ldots, m\}$, i.e. $\left.i i\right)$ holds.

Let $x^{\prime}=f\left(x_{m+1}^{1}\right)$. Observe that the vertex $x_{m+1}^{1}$ has also exactly $k$ neighbors outside $H_{m+1}^{1}$ (in contrast with vertices $x_{1}^{1}, \ldots, x_{m}^{1}$ that might have further neighbors outside $F$ ), the vertices $v_{1}^{1}, \ldots, v_{k}^{1}$ must be mapped bijectively onto the $k$ neighbors of $x^{\prime}$. Hence $N_{H}\left(f\left(x_{i}^{1}\right)\right)=N_{H}\left(x^{\prime}\right)$ for each $i$, i.e. $\left.i i i\right)$ holds.

Consequently, the restriction of $f$ to $F$ is 2 -fold on the vertices $x_{1}^{1}, \ldots, x_{m}^{1}$, i.e. a quasi-covering and $i$ ) holds as well. This argument concludes the proof that $F$ with specified vertices $u_{1}, \ldots, u_{m}$ is a coloring gadget for $H$.

\section{The NP-hardness reduction}

Recall that for a fixed graph $H$ the problem $H$-QCOVER is defined as follows:

Problem: $H$-QCOVER

Input: A graph $G$

Query: Does $G$ allow a quasi-covering to $H$ ?

Note that for all graphs $H$ the problem $H$-QCover belongs to the class NP, since the properties of a quasi-covering can be verified in polynomial time.

In order to prove Theorem 1 we distinguish several cases according to the structure of the graph $H$. We first show an NP-hardness reduction from the following well-known NP-complete problem [10, problem LO6]:

Problem: 2-IN-4 SAT

Input: A formula $\Phi$ in CNF where every clause contains exactly four literals Query: Could $\Phi$ be satisfied such that every clause contains exactly two positively valued literals?

Since 2-IN-4 SAT is the only version of SAT problem we use, we reserve the word satisfiable for formulas which are 2-in-4 satisfiable.

Lemma 4. Let $H$ be a connected graph on at least three vertices. If $H$ has a maximal distance vertex $x \in V(H)$ of degree two or if all maximal vertices of $H$ are of degree one and some maximal vertex has neighbor $x$ of degree two, then the H-QCOVER problem is NP-complete.

Proof. Let $\Phi$ be an instance of 2-IN-4 SAT. Denote the clauses of $\Phi$ by $C_{1}, \ldots, C_{m}$ and its variables by $v_{1}, \ldots, v_{n}$. We construct a graph $G_{\Phi, H}$ as follows:

We start with a disjoint union of a copy of the coloring gadget $\mathrm{CG}_{H}(x, n)$ with specified vertices $u_{1}, u_{2}, \ldots, u_{n}$ and a copy of $\mathrm{CG}_{H}(x, 2 m)$ with specified $w_{1}, w_{1}^{\prime}, w_{2}, w_{2}^{\prime}, \ldots, w_{m}, w_{m}^{\prime}$. The existence of these gadgets is guaranteed by Lemmata 2 and 3 . Then we include extra $2 n$ new vertices $p_{1}, q_{1}, p_{2}, q_{2}, \ldots, p_{n}, q_{n}$ and connect each vertex $u_{i}$ with vertices $p_{i}$ and $q_{i}$.

If any variable $v_{i}$ is one of the positive literals of $C_{j}$, then we join $w_{j}$ with $p_{i}$ and also $w_{j}^{\prime}$ with $q_{i}$. As a counterpart, if $\neg v_{i} \in C_{j}$ then we insert edges $w_{j} q_{i}$ and $w_{j}^{\prime} p_{i}$. This step concludes the construction of $G_{\Phi, H}$ (see Figure 6). 


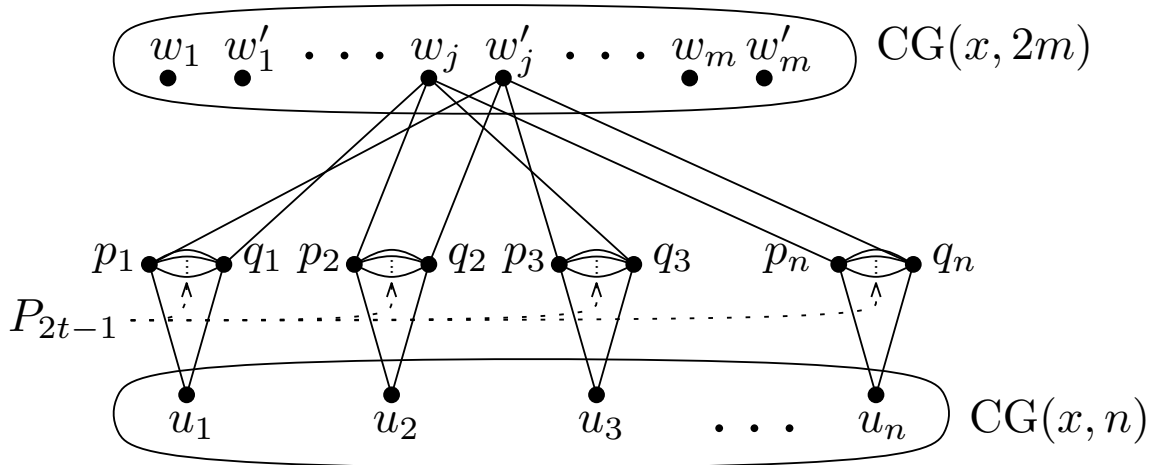

Fig. 6. An example of the construction of the graph $G$ for $H=P_{t}$. The edges depicted are related with the clause $C_{j}=\neg v_{1} \vee v_{2} \vee \neg v_{3} \vee v_{n}$. The graph $G_{\Phi, P_{t}}$ differs from $G$ only by the presence of the paths $P_{2 t-1}$.

Claim. If $G_{\Phi, H}$ is an induced subgraph of a quasi-cover $G$ of $H$ such that $\delta\left(G_{\Phi, H}\right) \subseteq\left\{p_{1}, q_{1}, \ldots, p_{n}, q_{n}\right\}$, then $\Phi$ is satisfiable.

Suppose that $f: G \rightarrow H$ is a quasi-covering. The properties of both coloring gadgets yield that the images of both sets of specified vertices are in $H$ of degree 2 - the same as $\operatorname{deg}(x)$. Denote the two neighbors of $f\left(u_{1}\right)$ by $y$ and $z$.

Then we know that for all $i \in\{1, \ldots, n\}$ it holds that $\left\{f\left(p_{i}\right), f\left(q_{i}\right)\right\}=\{y, z\}$. Consequently, the vertices $y$ and $z$ are the two neighbors of each $f\left(w_{j}\right)$. We assign $v_{i}=$ true if and only if $f\left(p_{i}\right)=y$ (thus $v_{i}=$ false $\Longleftrightarrow f\left(p_{i}\right)=z$ ).

As $f$ restricted to each coloring gadget is a quasi-covering, it must be a quasi-covering also on the subgraph remaining after the removal of both gadgets except their boundaries. Therefore, two neighbors of each $w_{j}$ are mapped on $y$ and two of them on $z$. Since these neighbors correspond to literals in $C_{j}$, we know that there are exactly two positive literals in every clause $C_{j}$. Therefore, we have obtained the desired satisfying assignment and proved the claim.

Now we resume the proof of Lemma 4 and extend $G_{\Phi, H}$ into a graph $G$ such that $G$ quasi-covers $H$ if $\Phi$ is satisfiable.

According to Lemma 1 we construct a $2 d$-regular multi-quasi-cover of $H$ with a specified vertex $a$ and $d=\frac{\operatorname{lcmd}(H)}{2}$. Let $B$ be the graph resulting by the deletion of $a$ from the multi-quasi-cover.

We start the construction of $G$ with $d$ copies of $G_{\Phi, H}$. To obtain $G$, we then perform the following steps for each $i \in\{1, \ldots, n\}$ :

- First we determine $o_{i}$ to be the number of occurences of $v_{i}$ in $\Phi$.

- Then we insert in the so far constructed graph exactly $o_{i}+1$ copies of $B$.

- Now we identify $o_{i}+2$ sets, each of size $2 d$ : the first set consists of the copies of vertices $p_{i}$ and $q_{i}$ while the others are formed by neighbors of the deleted vertex $a$ in the $o_{i}+1$ copies of $B$.

- On this set system we build $2 d$ disjoint transversals ${ }^{1}$ and merge vertices of each transversal into a single vertex. (See Figure 7) In other words, we merge

${ }^{1}$ By a transversal of a set system $\mathcal{S}$ we mean the range of an injective map $\varphi: \mathcal{S} \rightarrow \bigcup \mathcal{S}$ such that $\forall S \in \mathcal{S}: \varphi(S) \in S$. 
$2 d\left(o_{i}+2\right)$-tuples of distinct vertices into $2 d$ single vertices, such that the boundary of each $G_{\Phi, H}$ and of each $B$ is preserved.

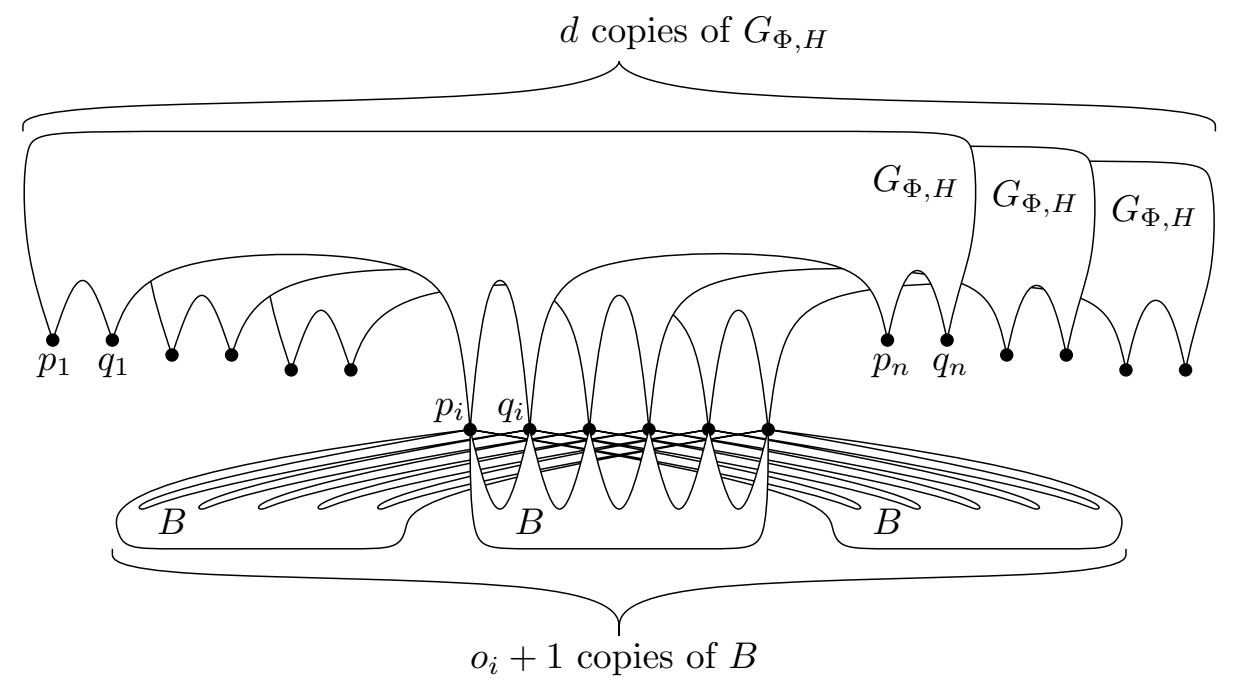

Fig. 7. The result of the $i$-th iteration, when $d=3$ and when variable $v_{i}$ has two occurences.

Suppose now that $\Phi$ is satisfiable. Let $y$ and $z$ be the neighbors of $x$. We define $f: V(G) \rightarrow V(H)$ as follows:

- $f\left(u_{i}\right)=f\left(w_{j}\right)=f\left(w_{j}^{\prime}\right)=x$ for all $i$ and $j$ in all $d$ copies.

- if $v_{i}=$ true then $f\left(p_{i}\right)=y$ and $f\left(n_{i}\right)=z$, otherwise $f\left(p_{i}\right)=z$ and $f\left(n_{i}\right)=y$;

- extend the so far defined $f$ to all copies of $B$ by Lemma 1 ;

- extend $f$ to a quasi-covering of all coloring gadgets of all $G_{\Phi, H}$ by Lemma 2 or 3 .

The obtained mapping is $\left(o_{i}+1\right)$-fold on each $N\left(p_{i}\right)$ and $N\left(q_{i}\right)$, hence a quasi-covering.

Note that for $H=P_{t}$ the above construction yields $d=1$ and $B=P_{2 t-1}$. Hence the graph $G$ consists from a single copy of $G_{\Phi, H}$, where each pair of vertices $p_{i}$ and $q_{i}$ is joined by $o_{i}+1$ paths of length $2 t-1$, as depicted in Figure 6 .

In the next case we reduce the following well known NP-complete problem[12]:

Problem: $k$-Chromatic Index

Input: A $k$-regular graph $D$

Query: Could the edges of $D$ be properly colored with $k$ colors, i.e. colors of adjacent edges are different?

Lemma 5. Let $H$ be a connected graph on at least three vertices. If $H$ has a maximal distance vertex $x \in V(H)$ of degree $k>2$ or if all maximal vertices of $H$ are of degree one and some maximal vertex has neighbor $x$ of degree $k>2$, then the $H$-QCOVER problem is NP-complete. 


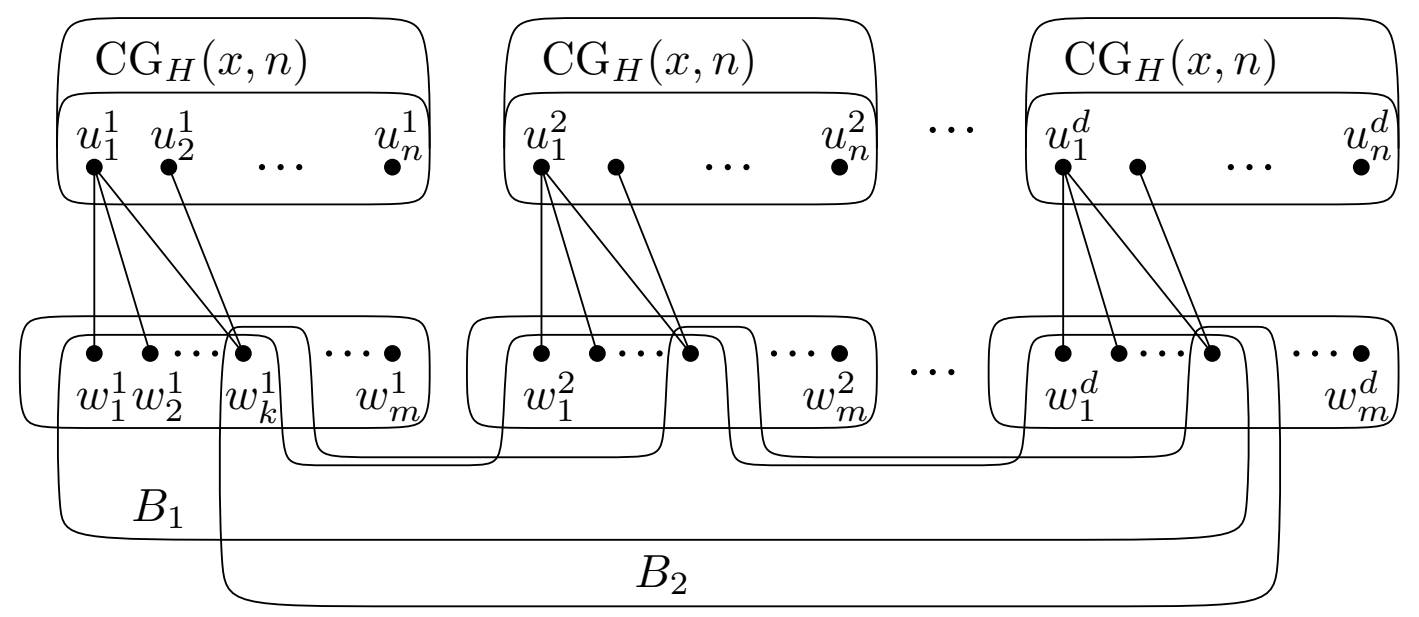

Fig. 8. Example of the construction of the graph $G$ for a $k$-regular graph $D$, where the vertex $v_{1}$ is incident with edges $e_{1}, e_{2}, \ldots, e_{k}$ and where $e_{k}=v_{1} v_{2}$.

Proof. We reduce the $k$-Chromatic InDEx problem using the neighborhood of $x$ as the set of colors. Let its instance be a $k$-regular graph $D$ on vertices $v_{1}, v_{2}, \ldots, v_{n}$ and edges $e_{1}, e_{2}, \ldots, e_{m}$. Let also $d=\frac{\operatorname{lcmd}(H)}{k}$.

We take $d$ copies of the graph $\mathrm{CG}_{H}(x, n)$ and denote its specified vertices $u_{1}^{1}, u_{2}^{1}, \ldots, u_{n}^{d}$. We also insert $d m$ new vertices $w_{1}^{1}, \ldots, w_{m}^{d}$ and make each $w_{j}^{t}$ adjacent to $u_{i}^{t}$ whenever $v_{i}$ is incident with $e_{j}$ in $D$.

According to Lemma 1 we construct a $k d$-regular multi-quasi-cover of $H$ with a specified vertex $a$. Let $B$ be the graph resulting by the deletion of $a$ from the multi-quasi-cover.

For every vertex $v_{i}$ of $D$ we perform the following two steps. We first identify the neighbors of $u_{i}^{1}, \ldots, u_{i}^{d}$ among vertices $w_{1}^{1}, \ldots, w_{m}^{d}$. As $D$ is $k$-regular, this selection provides a set of size $k d$. Then we take an extra copy $B_{i}$ of $B$, indentify the $k d$ former neighbors of the deleted vertex $a$, and merge them in arbitrary bijective manner with the $k d$ vertices selected in the previous step . This construction leads to $G$, the instance of the $H$-QCover problem (see Figure 8).

We claim that $G$ quasi-covers $H$ if and only if the edges of $D$ can be properly colored with $k$ colors. For the forward implication suppose that $f: G \rightarrow H$ is a quasi-covering. The properties of coloring gadget $\mathrm{CG}_{H}(x, n)$ imply that all neighbors of each $u_{i}^{1}$ among $w_{1}^{1}, \ldots, w_{m}^{1}$ are mapped by $f$ bijectively to the neighbors of $f\left(u_{1}^{1}\right)$. We define a proper edge $k$-coloring $c: E(D) \rightarrow N_{H}\left(f\left(u_{1}^{1}\right)\right)$ of the graph $D$ by $c\left(e_{j}\right)=f\left(w_{j}^{1}\right)$.

For the opposite implication suppose that $c: E(D) \rightarrow N_{H}(x)$ is a proper $k$-edge coloring of $D$. We define a mapping $f$ such that $f\left(u_{i}^{t}\right)=x$, and also $f\left(w_{j}^{t}\right)=c\left(e_{j}\right)$ for all $i, j$ and $t$.

We then extend $f$ to a quasi-covering of the whole graph $G$. The existence of these extensions is guaranteed by the properties of coloring gadgets (on each copy of $\mathrm{CG}_{H}(x, n)$ ), and also by Lemma 1 (on each copy of $B$ ). To the latter case we note that the partial mapping $f$ on any $\delta\left(B_{i}\right)$ corresponds to the mapping $\varphi$ of Lemma 1. 
Consider now any vertex $w_{j}^{t}$ that corresponds to a vertex $w$ of the multiquasi-cover. Suppose that the quasi-covering obtained by the extension of $\varphi$ is $c$-fold on $N(w)$; in fact $c=\frac{d k}{\operatorname{deg}(\varphi(w))}$. Then, since $w_{j}^{t}$ is on the boundary of two copies of $B$, we get that $f$ is $2 c$-fold on $N\left(w_{j}^{t}\right)$. By this argument we may conclude that $f$ is a quasi-covering.

Lemmas 4 and 5 constitute the NP-hardness part of the proof of Theorem 1. The polynomial part is straightforward: only edgeless graphs quasi-cover $P_{1}$; while a graph quasi-covers $P_{2}$ if and only if it is bipartite without isolated vertices. Both these classes could be recognized in linear time.

\section{Conclusion}

We have proved the dichotomy for the computational complexity of $H$-QCOVER problem when the graph $H$ is connected. This can be combined with a construction of Fiala and Paulusma [9, Proposition 5] to get a classification also for disconnected simple graphs:

Corollary 3. The $H$-QCOVER problem is polynomially solvable if either $H$ is edgeless or if $H$ is bipartite and at least one of its components is isomorphic to $K_{2}$. Otherwise, it is NP-complete.

The construction provides a quasi-cover of a chosen component, while it forbids all locally surjective homomorphisms to other components. Note that the other constructions presented in that paper do not provide quasi-covers, so our classification of connected graphs was a key ingredient for Corollary 3.

The classification is open for multigraphs with possible semiedges; these appear naturally in the topological models.

\section{References}

1. J. Abello, M. R. Fellows and J. C. Stillwell: On the complexity and combinatorics of covering finite complexes, Australian Journal of Combinatorics 4 (1991), 103-112.

2. O. Bílka, B. Lidický, M. Tesař: Locally injective homomorphism to the simple Weight graphs, Theory and Applications of Models of Computation, TAMC 2011, LNCS 6648, 471-482.

3. H. L. Bodlaender: The classification of coverings of processor networks, Journal of Parallel Distributed Computing 6 (1989), 166-182.

4. J. Fiala, and J. Kratochvíl: Complexity of partial covers of graphs, In Algorithms and Computation, ISAAC (2001), LNCS 2223, 537-549.

5. J. Fiala, and J. Kratochvíl: Partial covers of graphs, Discussiones Mathematicae Graph Theory 22 (2002), 89-99.

6. J. Fiala, J. Kratochvíl and A. Pór: On the computational complexity of partial covers of Theta graphs, Discrete Applied Mathematics 156 (2008), 1143-1149.

7. J. Fiala, and J. Kratochvíl: Locally injective graph homomorphism: Lists guarantee dichotomy, Graph-Theoretical Concepts in Computer Science, WG (2006), LNCS 4271, 15-26. 
8. J. Fiala and J. Kratochvíl: Locally constrained graph homomorphisms - structure, complexity, and applications, Computer Science Review 2 (2), (2008), 97-111.

9. J. Fiala and D. Paulusma: A complete complexity classification of the role assignment problem, Theoretical computer science 349 (1), (2005), 67-81.

10. M. R. Garey and D. S. Johnson: Computers and Intractability: A Guide to the Theory of NP-Completeness W. H. Freeman \& Co Ltd (1979).

11. P. Hell and J. Nešetřil: On the complexity of H-colouring, Journal of Combinatorial Theory, Series B 48 (1990), 92-110.

12. I. Holyer: The NP-completeness of edge-coloring: SIAM Journal on Computing 10 (4), (1981), 718-720.

13. J. Kratochvíl, A. Proskurowski and J. A. Telle: Covering regular graphs, Journal of Combinatorial Theory B 71 (1997), 1-16.

14. J. Kratochvíl, A. Proskurowski and J. A. Telle: Covering directed multigraphs I. colored directed multigraphs, In Graph-Theoretical Concepts in Computer Science, WG (1997), LNCS 1335, 242-257.

15. J. Kratochvíl, A. Proskurowski and J. A. Telle: Complexity of graph covering problems, Nordic Journal of Computing 5 (1998), 173-195.

16. P. Kristiansen and J. A. Telle: Generalized H-coloring of graphs. In Algorithms and Computation, ISAAC 01 (2000), LNCS 1969, 456-466.

17. B. Lidický and M. Tesař: Locally injective homomorphism to the Theta graphs, International Workshop on Combinatorial Algorithms, IWOCA 10 (2010), LNCS 6460, 326-336.

18. J. Matoušek and J. Nešetřil: Invitation to Discrete Mathematics, Oxford University Press, (2008). 